\title{
Final Results of the Efficient TerraSAR-X Calibration Method
}

\author{
M. Schwerdt, B. Bräutigam, M. Bachmann, B. Döring, Dirk Schrank and Jaime Hueso Gonzalez \\ Microwave and Radar Institute of the German Aerospace Center (DLR) \\ P.O.-Box 1116, D-82230 Weßling, Germany \\ phone: + (49) 0815328-3533, fax: + (49) 0815328-1449, email: Marco.Schwerdt@dlr.de
}

\begin{abstract}
TerraSAR-X is a satellite mission for scientific and commercial applications operating a highly flexible X-band SAR instrument with a multitude of different operation modes. As product quality is of crucial importance, the success or failure of the mission depends essentially on the method of calibrating TerraSAR-X in an efficient way during commissioning the entire system in a restricted time. Only then, product quality and the correct in-orbit operation of the entire SAR system can be ensured. The paper describes both the in-orbit calibration method for TerraSAR-X and dedicated activities performed during the commissioning phase as well as final results derived from all calibration procedures.
\end{abstract}

Index Terms-Calibration, Antenna Model, TerraSAR-X System.

\section{INTRODUCTION}

On June 15th, 2007, the first German SAR satellite for commercial and scientific applications, TerraSAR-X, was launched. The essential task of calibrating TerraSAR-X is to estimate and correct systematic error contributions throughout the complete SAR system and to tie-down image information (magnitude and phase) to reference units in geophysical terms. The quality of this calibration process depends on the inherent stability of the radar system and the capability to determine and monitor the radiometric and geometric characteristics [1].

TerraSAR-X is designed to cover a wide range of swath positions and to operate in different operation modes [2] by switching the instrument over a multitude of different antenna beams (more than 12,000). Due to this high degree of flexibility of TerraSAR-X (StripMap, Spotlight, ScanSAR, right/left looking, etc.) and a tight performance with an absolute radiometric accuracy better than $1 \mathrm{~dB}$ it becomes clear, that a conventional calibration approach is not feasible. The real measurement of all antenna beams in all operation modes, as performed for the ASAR instrument of ENVISAT across the rainforest [3] is to time-consuming. Hence, a new, more efficient, and affordable calibration concept was developed [4], [5]. The key element of this calibration concept is a novel antenna model approach [6].

Furthermore, to account for the restricted time of calibration campaigns performed during the five months commissioning phase (CP) of TerraSAR-X, the number of passes and places of test sites is optimized versus cost and time effort by calibrating several beams and polarization modes with the same test site [7].
The paper describes both in-orbit calibration procedures and final results derived from all measurements performed for calibrating TerraSAR-X during the CP.

\section{SCOPE AND STRATEGY}

In addition to the complexity of TerraSAR-X and the corresponding challenge described before, the main goal after launch of the satellite is to provide calibrated and verified SAR data products as soon as possible. Thus, a strategy for an efficient but robust calibration method was developed. Applying the novel antenna model approach the inorbit calibration method can be sub-divided into six major tasks performed during commissioning of TerraSAR-X. The successive baseline calibration procedures are:

1) Geometric Calibration, to assign the SAR data to the geographic location on the earth's surface.

2) Antenna Pointing Determination, to obtain a correct beam pointing of the antenna.

3) Antenna Model Verification, to ensure the provision of the antenna patterns of all operation modes and the gain offset between different beams.

4) Relative Radiometric Calibration, for the radiometric correction of SAR data within an illuminated scene.

5) Absolute Radiometric Calibration, for measuring the SAR system against standard targets with well known radar cross section (RCS).

However, the success of performing all these activities within a restricted period of time for commissioning TerraSAR-X is essentially dependent on the stability of the instrument. Thus, an accurate facility for the Internal Calibration (the 6th major task) had to be established and implemented for monitoring and compensating drift effects of the instrument.

Results of calibrating TerraSAR-X in-orbit are described in the following.

\section{INTERNAL CALIBRATION}

Temperature drifts and internal hardware characteristics influence the radar signal path causing gain and phase fluctuation during data acquisition. For monitoring and compensating these effects the radar instrument of TerraSAR-X hosts an internal calibration facility comprising both conventional internal calibration pulses and the so called PN-gating method, a further novel calibration method [8], [9]. 


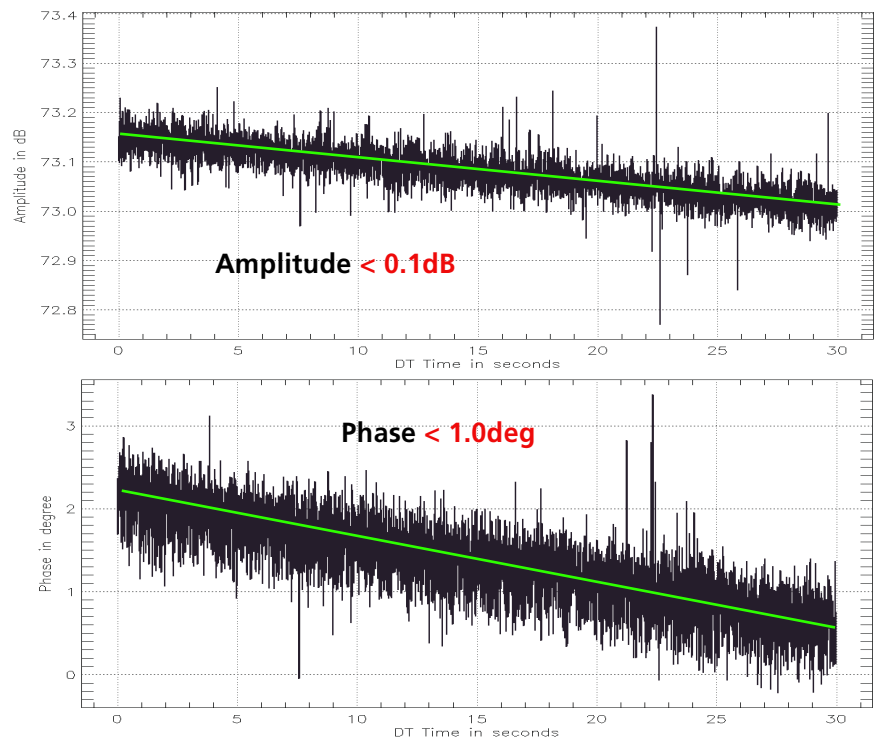

Fig. 1. Residual drift error of the instrument by applying the interna calibration facility, black lines: radar pulses wihtin a datatake, green line fit derived from calibration pulses.

The amplitude and the phase drift of the TerraSAR-X instrument within a data take are shown in Fig. 1, whereby the black lines are the radar pulses and the green line is a fit derived from calibration pulses activated before and after the data take, i.e the proper radar pulses. Thus, by monitoring the instrument not only by telemetry data (e.g. the temperature measured inside the instrument during operation) but also by internal calibration pulses even small drift effects can be compensated for down to an accuracy $<0.1 \mathrm{~dB}$ for the amplitude and better than 1 deg for the phase.

Furthermore, the novel PN-Gating method, developed and established for characterizing individual transmit/receive (T/R) modules in operation of an active antenna array [8], has now been successfully verified for the first time in orbit by TerraSAR-X [9]. As shown in Fig. 2 the actual amplitude setting of each $T / R$ module can be measured down to an accuracy of $0.2 \mathrm{~dB}$ and better than $2 \mathrm{deg}$ for the phase setting.

Based on this accurate internal calibration facility the different calibration procedures described in section II could be performed within the tight schedule of commissioning TerraSAR-X.

\section{Geometric CAlibration}

The purpose of geometric calibration is the geometric assignment of the SAR system to the earth's surface. Two major effects influence the correct localization of the product:

- the internal electronic delay of the instrument and

- range offset/propagation delay by hydrostatic effects.

Measuring the TerraSAR-X system against passive targets with no internal delay and consequently no additional source of error these effects could be determined and compensated for. But also the annotation of the source packets is checked against systematic azimuth or range line shifts respectively.

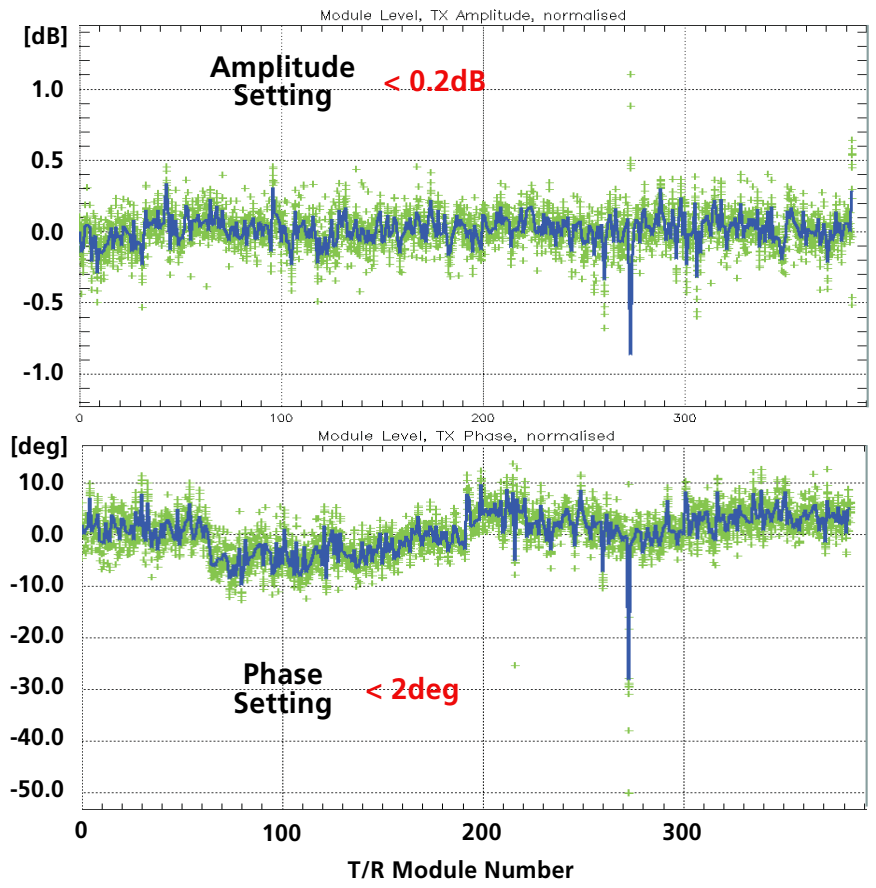

Fig. 2. In-flight measured excitation coefficient of $384 \mathrm{~T} / \mathrm{R}$ modules of the TerraSAR-X instrument by applying the so called PN-gating method.

The residual slant range offset as function of look angle is shown in Fig. 3. Thus, for TerraSAR-X a pixel localization accuracy of $30 \mathrm{~cm}$ could be achieved, i.e. the connection between the SAR data and the earth surface could be calibrated down to an accuracy of almost one magnitude better than the product specification with $2 \mathrm{~m}$.

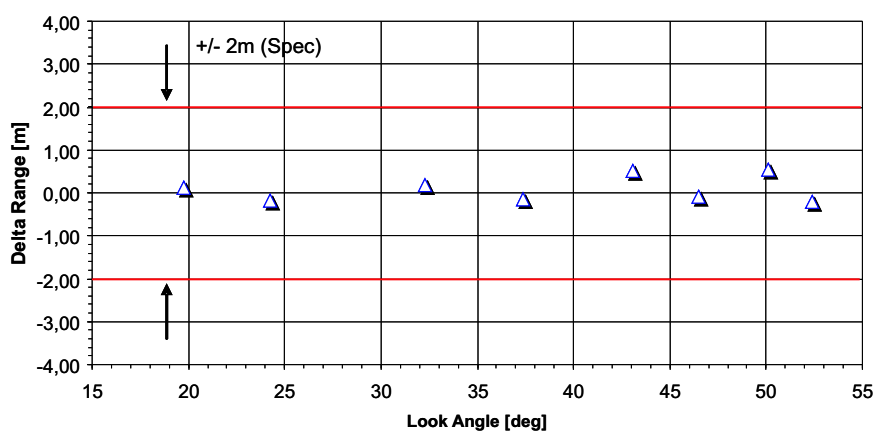

Fig. 3. Residual slant range offset in dependence of the look angle after correcting for instrument delay and propagation effects.

By this accurately calibrated TerraSAR-X system the impact of propagation effects on the range delay could be verified for the first time by a SAR system, as shown in Fig. 4. The theoretical path extension caused by tropospheric refraction along the hydrostatic path and well known from the global positioning system (GPS) is depicted by the pink line.

\section{Antenna Pointing}

An important task is the determination of beam pointing errors coming from mechanical and electrical antenna misspointing as well as attitude control offsets. These errors are 


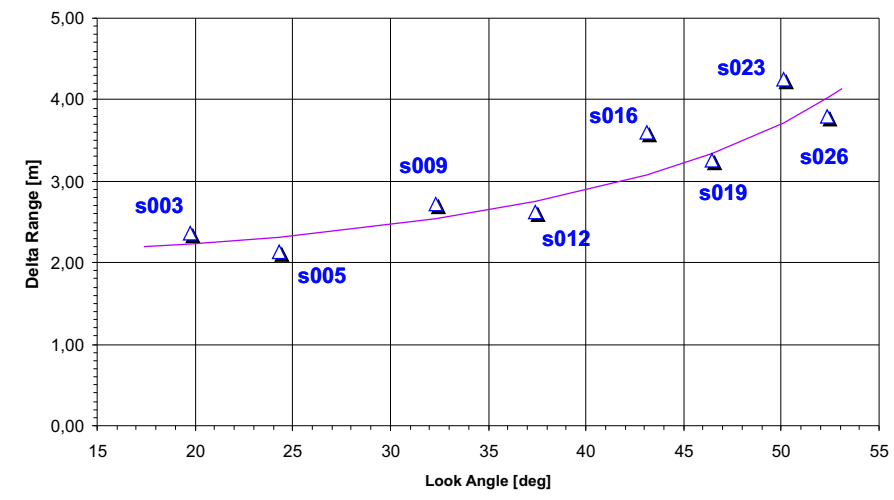

Fig. 4. Range offset derived from the range delay and measured for different beams of TerraSAR-X (triangles). The theoretical path extension by hydrostatic effects is depicted by the pink line.

measured in elevation and in azimuth using an appropriate antenna pattern over rain forest and by ground receivers. The patterns used feature a notch in the mid of the pattern, i.e. at Odeg and consequently an offset of this notch indicates a squint angle of the antenna. Driven by the radiometric accuracy budget a pointing knowledge of $0.015 \mathrm{deg}$ in elevation and $0.002 \mathrm{deg}$ in azimuth had to be achieved for TerraSAR-X. One example of measuring the beam pointing in elevation is shown in Fig. 5 by illuminating the Amazone rainforest with a notch pattern.

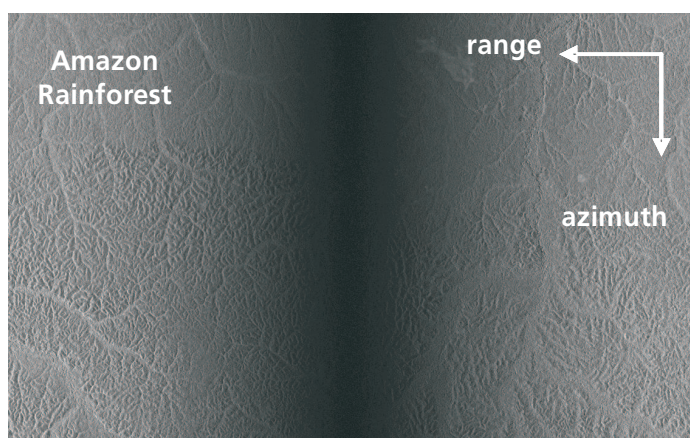

Fig. 5. Amazon rainforest illuminated by a notch pattern in StripMap operation of TerraSAR-X.

As expected, the brightness in the mid of the scene is reduced due to the notch within the elevation pattern. Deriving the gamma profile of such data takes and applying the corresponding reference pattern derived from the antenna model, the offset of the notch and consequently the actual pointing of the TerraSAR-X antenna in elevation could be determined down to an accuracy better than the required $0.015 \mathrm{deg}$, as shown in Fig 6.

The beam pointing in flight direction could be determined likewise by notch patterns now operated in azimuth direction and measured by deployed ground receivers. The achieved pointing knowledge in azimuth is better than the required $0.002 \mathrm{deg}$ as well.

Hence, by readjusting the attitude of the satellite the detected miss-pointing of the antenna beam could be removed

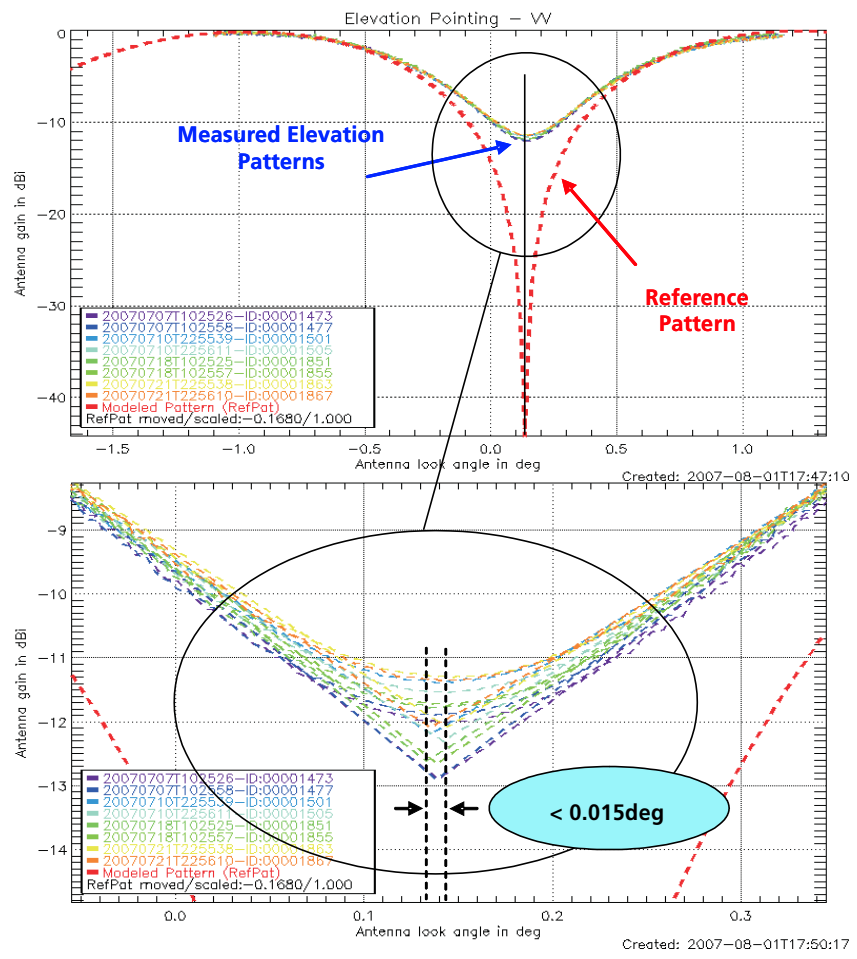

Fig. 6. Beam pointing in elevation of TerraSAR-X determined by measured notch patterns across the rainforest.

and the remaining squint angle is in the order of the required pointing knowledge in both directions, i.e. negligible.

\section{Vi. Antenna Model Verification}

The characterization of the antenna is based on a precise antenna model, which was developed before launch considering different input parameters like:

- the geometry of the antenna,

- the excitation coefficients of all 384 T/R modules,

- a drift and failure matrix and

- embedded radiation patterns measured on ground from single sub-arrays of the antenna.

This antenna model was validated against near field patterns precisely measured on-ground before launch. This validation was performed successfully showing the excellent performance of the antenna model [6].

To even verify the antenna model performance for the whole antenna in space, in-orbit measurements were performed during the commissioning phase. This verification was performed with a few selected beams really measured in-flight and can be divided into three main tasks:

- measurements across the rainforest to verify the elevation pattern shape,

- ScanSAR measurements over rainforest to verify the calculated vs. simulated peak-to-peak gain offset between different beams and

- measurements using ground receivers to verify azimuth pattern shape. 


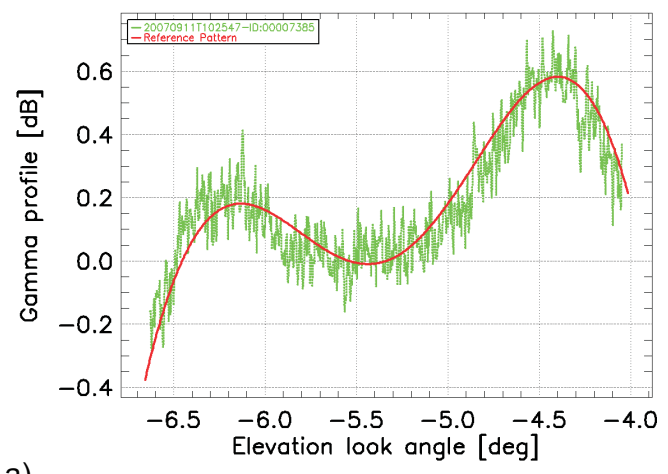

a)

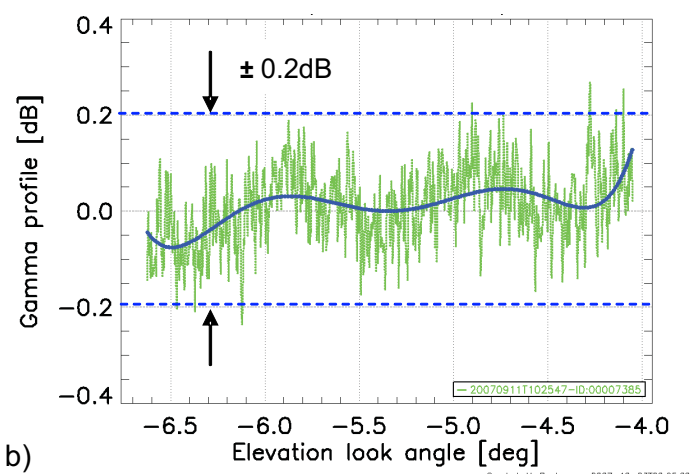

Fig. 7. a) Comparison between the gamma profile (green points) derived from a rain forest scene and the reference elevation pattern (red line), b)difference between reference pattern and gamma profile, the blue line is a fit of this difference.

For the pattern shape in elevation, simulated antenna patterns were compared with estimated patterns derived from SAR data acquired over Amazon rainforest. As Amazon rainforest is a quite homogeneous scatterer, the pattern shape is clearly visible in the SAR raw data. However, for an accurate estimation of the pattern shape, the images have to be masked out from disturbing image areas like rivers or de-forested areas [10]. Then, all range lines are summed up to obtain the so called gamma profile, as shown in Fig. 7a) by the slight noisy green curve, and compared to the modeled reference pattern (red line). The verification of the antenna model in elevation shows a very good accordance between the simulated antenna pattern and the measured gamma profiles. The deviation and hence the accuracy of the antenna model is below $+/-0.2 \mathrm{~dB}$ (peak-to-peak), which is excellent, as can be seen for the exemplary gamma profile in Fig. 7b).

The verification of the antenna patterns in azimuth direction was performed for transmit patterns using ground receivers. One receiver measures the amplitude of the pulses transmitted by the TerraSAR-X satellite during an overflight. Transformed to the antenna elevation angles and corrected by position information, the azimuth antenna pattern is obtained and can be compared to the modeled pattern. This is shown in Fig. $8 \mathrm{a}$ ), where the measured pattern (colored) are compared to the reference pattern (red). As same as for elevation, the antenna model verification shows remarkable results for azimuth pattern. Even for the "double squinted" beam (azimuth
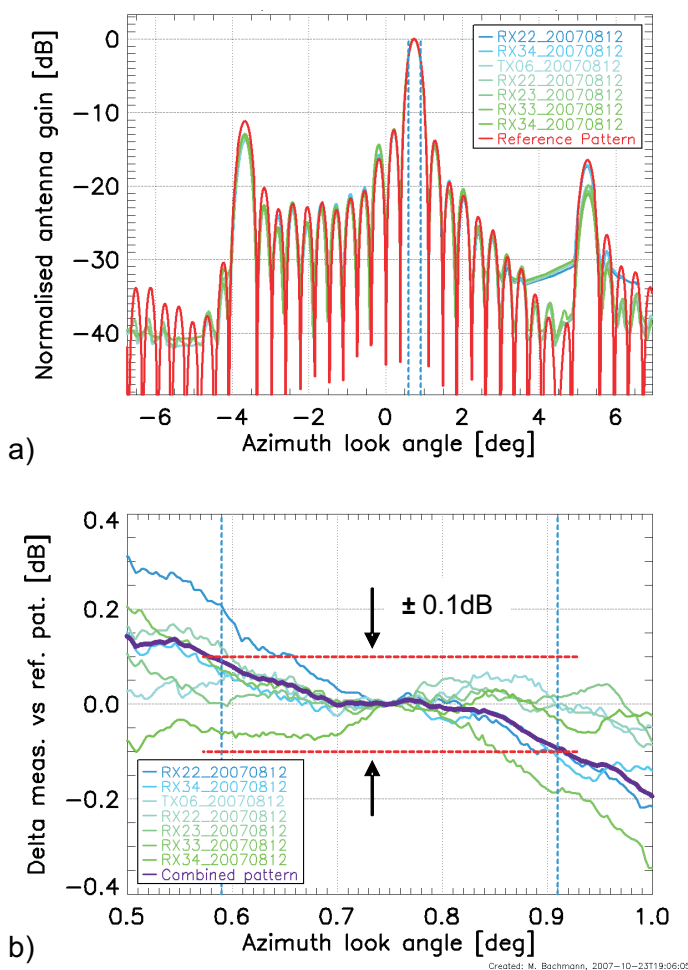

Fig. 8. a) Transmit pattern measured by ground receivers (colored) and compared to a reference pattern (red), b) purple line: difference between measured pattern averaged over all ground receiver measurements and the reference pattern.

steering: $0.75 \mathrm{deg}$, elevation steering $-16.5 \mathrm{deg}$ ) shown here, the resulting deviation within the $3 \mathrm{~dB}$ beamwidth is less than +/-0.1 dB (peak-to-peak).

The beam-to-beam gain offset prediction i. e. the prediction of the variation of the gain in the maximum relative from beam to beam in elevation, is verified evaluating ScanSAR data. In ScanSAR acquisitions, the beam is switched sequentially from burst to burst between four neighbouring swaths to get a broader swath width than for normal Stripmap acquisitions. In Level1B-Products, these four subswaths are still separated but are combined in higher level products. By generating the unnormalised gamma profile for each of the four subswaths, the relative gain deviation can be determined. Thus, the antenna model could be verified once again with an accuracy below $+/-0.2 \mathrm{~dB}$, now with respect to the gain offset between different beams, as shown for the exemplary result in Fig. 9.

The verification in flight shows that the maximum deviation between the antenna model and the measurements for both the shape within the main-beam and the gain-offset between different beams is less than $+/-0.2 \mathrm{~dB}$ (peak-to-peak). Hence, by this precise antenna model approach the time and the effort for the relative and the absolute radiometric calibration could be reduced extremely because only a few selected beams had to be really measured in-flight. 

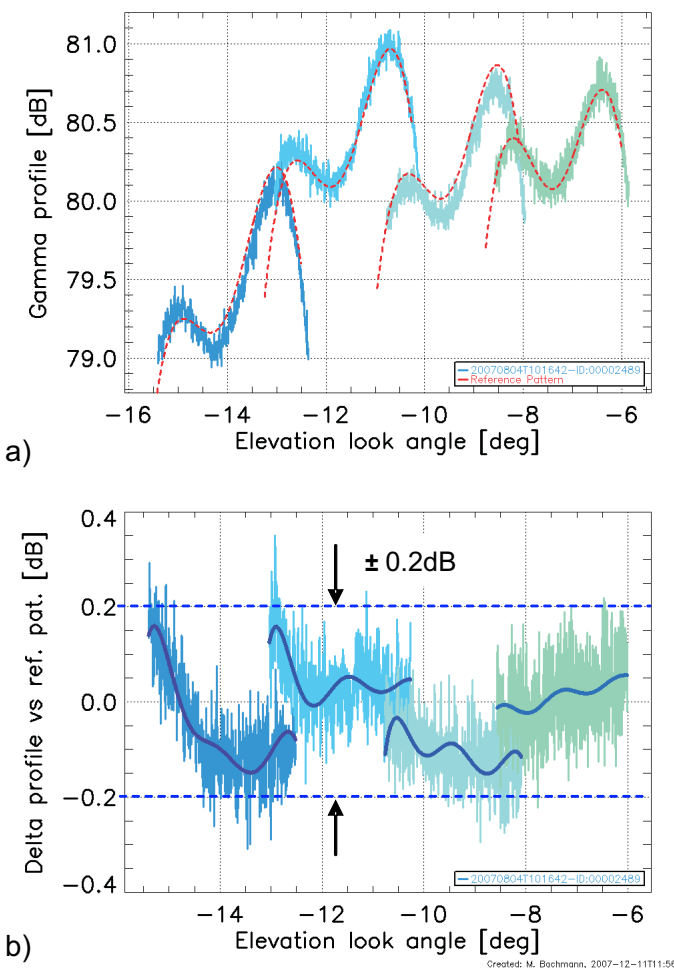

Fig. 9. a) Beam-to-beam gain offset verification comparing ScanSAR Beams measured (colored) and derived by the model (red), b) difference between reference and measured pattern, whereby the blue lines are fits of the difference.

\section{RELATIVE AND ABSOLUTE RADIOMETRIC CALIBRATION}

After the successful in-orbit verification of the antenna model thousands of beam patterns required for the relative radiometric correction during SAR data processing can be derived by the antenna model for all operation modes and all incidence angles. In order to further reduce the calibration effort, the absolute radiometric calibration is likewise based on the antenna model, i.e. real measurements over point targets with known radar cross section are performed on a reduced set of beams. The absolute calibration factor of all other beams and modes are derived via the antenna model by applying the beam-to-beam gain prediction.

One example of the absolute calibration factor derived from reference targets deployed across an illuminated swath is shown in Fig. 10. Separated according to the position of each target (near, mid and far position within the swath) and concentrating on the mean value of all measurements at one position, the variation within the swath is less than $+/-0.2 \mathrm{~dB}$. Consequently, the verification of the antenna model could be performed once again, now by a real end-to-end system test with deployed reference targets. However, the standard deviation of all measurements within one scene or across the whole swath defines the relative radiometric accuracy and for TerraSAR-X we have achieved $0.3 \mathrm{~dB}$ in StripMap operation.

In the next step we were interested whether the gainoffset between different beams could be likewise verified

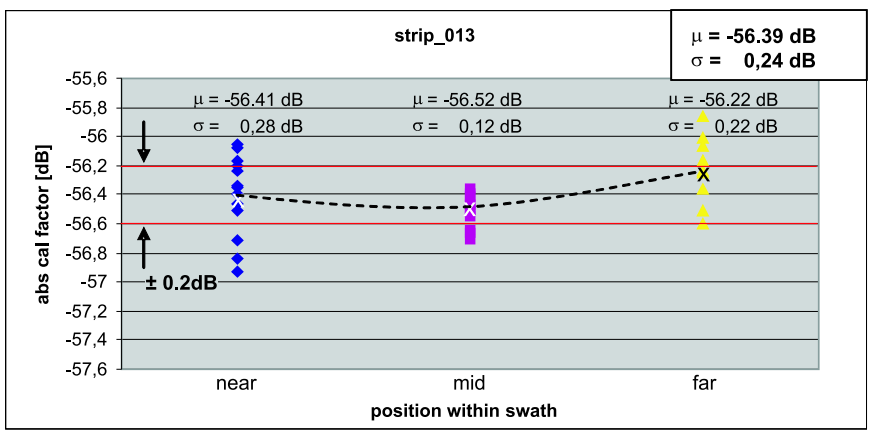

Fig. 10. Absolute calibration factor derived from reference targets deployed across the swath in StripMap operation.

by deployed reference targets. For this purpose the absolute calibration factor derived from all measurements was separated according to the beam ID, i.e. as function of look angle, as shown in Fig. 11. For this purpose only targets deployed in the mid of the scene were considered in order to avoid the impact of the shape of the pattern across the swath. And again, concentrating on the mean value of all measurements for one look angle, we could achieve a maximum variation of the gain offset between different beams of less than $+/-0.2 \mathrm{~dB}$ by deployed reference targets.

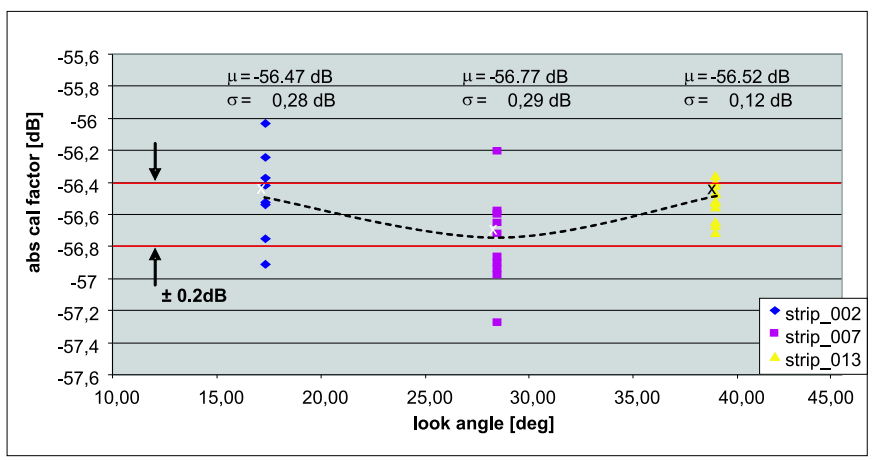

Fig. 11. Absolute calibration factor versus beam ID/look angle derived from reference targets deployed in the mid of the corresponding scene.

Hence, absolute radiometric calibration of the whole TerraSAR-X system must be independent on both, the position of the targets deployed within the swath and the beam being operated. Because the shape of the antenna pattern and the gain-offset between different beams are already compensated by applying the antenna model during SAR data processing. And indeed, displaying the absolute calibration factor derived from all measurements only as function of measurement ID, as shown in Fig. 12, no significant dependency can be observed. Consequently, the absolute radiometric accuracy is defined by the standard deviation of all measurements. For TerraSAR-X we have achieved an absolute radiometric accuracy of $0.31 \mathrm{~dB}$ for StripMap operation during the CP of TerraSAR-X.

However, considering a conservative assumption for the long term stability of the instrument, an absolute radiometric accuracy of $0.6 \mathrm{~dB}$ for StripMap Basic Products is ensured dur- 


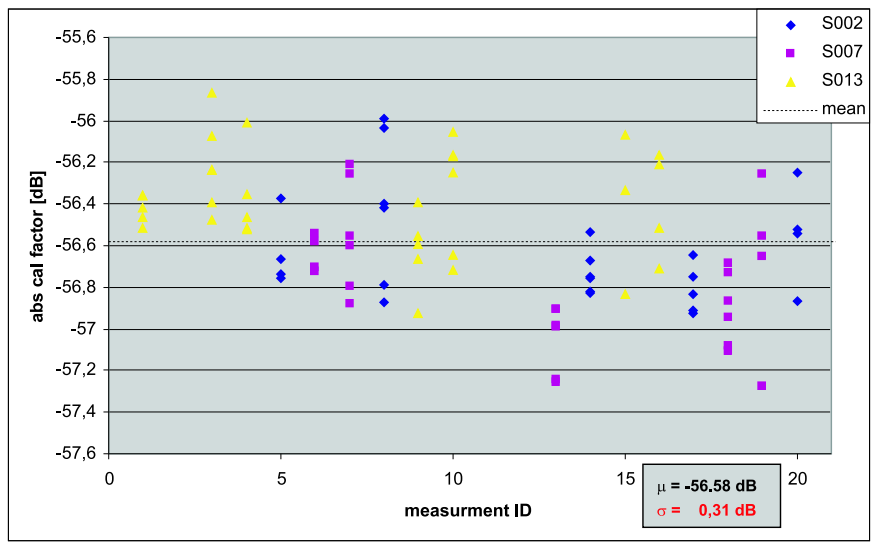

Fig. 12. Absolute calibration factor versus measurement ID derived from all reference targets deployed and all StripMap beams being measured.

ing the lifetime of TerraSAR-X. Thus, a whole SAR system at a distnace of more than $500 \mathrm{~km}$ could be successfully adjusted and calibrated with the accuracy of laboratory equipment.

\section{CONCLUSION}

The results of calibrating TerraSAR-X approve the accuracy calculated before launch and put the described strategy to calibrate efficiently a multiple mode SAR system like TerraSAR-X on a solid base. The key element of this strategy is an antenna model approach shifting most of the antenna characterization effort from the commissioning phase to pre-launch activities. TerraSAR-X is the first SAR satellite calibrated with this innovative approach and all calibration procedures could be successfully performed within the given time of commissioning TerreSAR-X.

For this purpose, a calibration facility comprising reliable and accurate ground calibration hardware [11] as well as software tools for evaluating all measurements was developed and established by the Microwave and Radar Institute of DLR. Furthermore a calibration field in South Germany enclosing an area of $120 \mathrm{~km} \mathrm{x} 40 \mathrm{~km}$ and comprising 30 target positions was deployed and operated during the commissioning phase. It has been shown that all calibration systems are working very well. The stability and accuracy of the system and especially the radar instrument itself is of unprecedented quality.

Tab. I lists the results of calibrating TerraSAR-X performed during the commissioning phase. It has to be mentioned that all requirements and/or goals have been achieved even better than predicted.

By this successful demonstration of the applied effective and exact calibration techniques a new benchmark has been settled not only for calibrating complex SAR systems but in principle for highly accurate spaceborne SAR sensors.

Keeping the costs affordable is the major challenge of calibrating future SAR sensors. e.g. like TanDEM-X [12] or Sentinel-1 [13], and the described calibration strategy using innovative methods like the applied antenna model approach and the PN-Gating method has made an important contribution to achieve this aim.

\begin{tabular}{|c|c|c|}
\hline Calibration Procedure & Goal & Achieved \\
\hline \multicolumn{3}{|l|}{ Internal Calibration } \\
\hline Amplitude & $0.25 \mathrm{~dB}$ & $\pm 0.2 \mathrm{~dB}$ \\
\hline Phase & $1 \mathrm{deg}$ & $\pm 1 \mathrm{deg}$ \\
\hline \multicolumn{3}{|l|}{ T/R Module Characterization } \\
\hline Amplitude & $0.3 \mathrm{~dB}$ & $<0.2 \mathrm{~dB}$ \\
\hline Phase & $2.5 \mathrm{deg}$ & $<2 \operatorname{deg}$ \\
\hline \multicolumn{3}{|l|}{ Antenna Pointing Knowledge } \\
\hline Elevation & $0.015 \mathrm{deg}$ & $<0.006 \mathrm{deg}$ \\
\hline Azimuth & $0.002 \mathrm{deg}$ & $<0.002 \mathrm{deg}$ \\
\hline \multicolumn{3}{|l|}{ Pixel Localization Accuracy } \\
\hline Azimuth & $2 \mathrm{~m}$ & $0.5 \mathrm{~m}$ \\
\hline Range & $2 \mathrm{~m}$ & $0.3 \mathrm{~m}$ \\
\hline \multicolumn{3}{|l|}{ Antenna Model Verification } \\
\hline Elevation Pattern & $\pm 0.2 \mathrm{~dB}$ & $< \pm 0.2 \mathrm{~dB}$ \\
\hline Azimuth Pattern & $\pm 0.1 \mathrm{~dB}$ & $< \pm 0.1 \mathrm{~dB}$ \\
\hline Beam-to-Beam Gain Offset & $\pm 0.2 \mathrm{~dB}$ & $< \pm 0.2 \mathrm{~dB}$ \\
\hline \multicolumn{3}{|l|}{ Radiometric Calibration } \\
\hline \multicolumn{3}{|l|}{ StripMap / ScanSAR } \\
\hline Radiometric Stability & $0.87 \mathrm{~dB}$ & $\pm 0.2 \mathrm{~dB} *$ \\
\hline Relative Radiometric Accuracy & $0.68 \mathrm{~dB}$ & $<0.3 \mathrm{~dB} / 0.4 \mathrm{~dB}$ \\
\hline Absolute Radiometric Accuracy & $0.9 \mathrm{~dB}$ & $<0.6 \mathrm{~dB} / 0.7 \mathrm{~dB}$ \\
\hline
\end{tabular}

TABLE I

Results of CALIBRATING TERRASAR-X

\section{REFERENCES}

[1] M. Schwerdt and D. Hounam, "Quality Control and Calibration of Future SAR Systems," in ODAS 2001, 3rd ONERA-DLR Aerospace Symposium, Paris, France, 2001, pp. S6-6.

[2] S. Buckreuss, R. Werninghaus, and W. Pitz, "German Satellite Mission TerraSAR-X," in 2008 IEEE Radar Confrerence, Rome, Italy, 2008.

[3] M. Zink and B. Rosich, "Antenna Elevation Pattern Estimation from Rain Forest Acquisitions," in ENVISAT/ASAR Calibration Review (ECR) of ESTEC, European Space Agency (ESA), Noordwijk, Netherland, 2002.

[4] M. Schwerdt, D. Hounam, J. L. Alvarez-Pérez, and T. Molkenthin, "The Calibration Concept of TerraSAR-X, a Multiple Mode High Resolution SAR," Canadian Journal of Remote Sensing, Vol. 31, No.1, Feb 2005.

[5] M. Schwerdt, D. Hounam, B. Bräutigam, and J. L. Alvarez-Pérez, "TerraSAR-X: Calibration Concept of a Multiple Mode High Resolution SAR," in 25th International Geoscience And Remote Sensing Symposium, Seoul, Korea, 2005.

[6] M. Bachmann, M. Schwerdt, B. Bräutigam, B. Grafmüller, A. Herschlein, and J. L. Alvarez-Pérez, "The TerraSAR-X Antenna Model Approach," in 2nd International ITG Conference on Antennas, Munich, Germany, 2007.

[7] M. Schwerdt, B. Bräutigam, M. Bachmann, and B. Döring, "TerraSAR$\mathrm{X}$ Calibration - First Results," in 26th International Geoscience And Remote Sensing Symposium, Barcelona, Spain, 2007.

[8] D. Hounam, M. Schwerdt, and M. Zink, "Active Antenna Module Characterisation by Pseudo-Noise Gating," in 25th ESA Antenna Workshop on Satellite Antenna Technology, Noordwijk, Netherlands, 2002.

[9] B. Bräutigam, M. Schwerdt, M. Bachmann, and M. Stangl, "Individual T/R Module Characterisation of the TerraSAR-X Active Phased Array Antenna by Calibration Pulse Sequences with Orthogonal Codes," in 26th International Geoscience And Remote Sensing Symposium, Barcelona, Spain, 2007.

[10] J. L. Alvarez-Pérez, M. Schwerdt, and M. Bachmann, "TerraSARX Antenna Pattern Estimation by Complex Treatment of Rainforest Measurements," in 26th International Geoscience And Remote Sensing Symposium, Denver, USA, 2006.

[11] B. Döring, M. Schwerdt, and R. Bauer, "TerraSAR-X Calibration Ground Equipment," in WFMN07, Conference of ITG - Commission on Wave Propagation, Dresden, Germany, 2007.

[12] J. Hueso Gonzalez, M. Bachmann, H. Fiedler, S. Huber, G. Krieger, and M. Zink, "DEM Calibration Concept of TanDEM-X," in 26th International Geoscience And Remote Sensing Symposium, Barcelona, Spain, 2007.

[13] E. Attema, "Mission Requirements Document for the European Radar Observatory Sentinel-1," ESA-ESTEC, ES-RS-ESA-SY-0007, July 2005 . 